\title{
Teaching quantum mechanics with the Hong-Ou-Mandel interferometer
}

Gustavo Armendáriz, Jorge Cravioto-Lagos, Victor Velázquez, Marcela Grether, Enrique López-Moreno, et al.

Gustavo Armendáriz, Jorge Cravioto-Lagos, Victor Velázquez, Marcela Grether, Enrique López-Moreno, E. J. Galvez, "Teaching quantum mechanics with the Hong-Ou-Mandel interferometer," Proc. SPIE 9289, 12th Education and Training in Optics and Photonics Conference, 928908 (17 July 2014); doi: 10.1117/12.2070280

EDent: 12th Education and Training in Optics and Photonics Conference, 2013, Porto, Portugal 


\title{
Teaching quantum mechanics with the Hong-Ou-Mandel interferometer
}

\author{
Gustavo Armendáriz ${ }^{a}$, Jorge Cravioto-Lagos ${ }^{a}$, Víctor Velázquez ${ }^{a}$, Marcela Grether ${ }^{a}$, Enrique \\ López-Moreno ${ }^{a}$ and E. J. Galvez ${ }^{b}$ \\ a Facultad de Ciencias, Universidad Nacional Autónoma de México, 04510 México, D.F., \\ México \\ ${ }^{b}$ Department of Physics and Astronomy, Colgate University, Hamilton, New York 13346.
}

\begin{abstract}
The Hong-Ou-Mandel interferometer is an optical device which allows us to prove experimentally the quantum nature of light via the quantum amplitude superposition of two indistinguishable photons. We have implemented this experiment as an advanced undergraduate laboratory experience. We were able to overcome well known difficulties with this experiment using recently reported techniques by Thomas et al. [Rev. Sci. Instr. 80, 036101 (2009), ].
\end{abstract}

Keywords: Quantum Optics, Hong-Ou-Mandel

\section{INTRODUCTION}

New demonstrations of fundamental aspects of quantum mechanics with single photons has led to an appreciation of the fundamental properties of quantum mechanics and their inclusion in quantum mechanics instruction. A series of experiments with single photons developed for the undergraduate laboratory has been a successful way to underscore these fundamentals by direct experimentation. Experiments such as the proof of the existence of the photon, ${ }^{1}$ the quantum eraser ${ }^{2}$ or quantum non-realism and nonlocallity ${ }^{3}$ deliver results that embody almost 100 years of discussions on the quantum nature of fundamental particles and light.

The subject of this article is twofold: First, to present the the implementation of a landmark experiment in quantum optics in the undergraduate laboratory: the Hong-Ou-Mandel (HOM) interference experiment. ${ }^{4}$ Second, to show in action the very important statement of the quantum mechanics: the multi-way behavior of the indivisible ${ }^{5}$ quantum photon (or particle with mass); in words of Feynman ${ }^{6}$ the only mistery of quantum physics.

We used this experiment in the context of our offering at graduate and advanced undergraduate physics courses on quantum optics. At the graduate level, it represents one of the possible special projects in a compulsory course (Laboratorio Avanzado) within the masters degree program, where the student not only treats fundamental quantum principles in an experiment, but also manipulates apparatuses and more sophisticated devices and technologies.

\section{QUANTUM DESCRIPTION OF A BEAM SPLITTER AND THE INTERFERENCE OF TWO PHOTONS}

The formalism of the two-path behavior of the one-photon-beam-splitter array can be considered a probabilistic rule that match with experimental observations, but don't offers a straightforward interpretation. Surely more than one teacher in classrooms, has been tempted to speak about of one photon that travels by two ways simultaneously. This interpretation is similar to the coexistence of states in atomic or nuclear physics, when we speak of the probability to find one electron (nucleon) in more than one allowed states. This multi-path state or coexistence of states is the heart of quantum mechanics. This represent the no-locality of the quantum states. One photon that interferes with itself or two indistinguishable photons that interferes destroying themselves selectively represent an evidence of such multi-way behavior. To observe this effect in the laboratory implies to observe the heart of the quantum world. ${ }^{7}$

We begin treating this problem by considering a single photon incident on a 50-50 beam splitter. If initially

12th Education and Training in Optics and Photonics Conference, edited by

Manuel F. P. C. Martins Costa, Mourad Zghal, Proc. of SPIE Vol. 9289, 928908

(C) 2014 SPIE, OSA, IEEE, ICO · doi: 10.1117/12.2070280

Proc. of SPIE Vol. 9289 928908-1 
one photon travels to the right but not "down", i. e. coming from above in Fig. (1), it can be described in the photon number representation by the Fock state, or photon occupation state:

$$
\left|\psi_{i}\right\rangle=|1\rangle_{R}|0\rangle_{D},
$$

where the subindices $R$ and $D$ refer to the mode of the light traveling to the right and down, respectively. In this problem the mode of the light where none is traveling "down" may appear trivial or unnecessary, but the use of the "vacuum" state $|0\rangle_{D}$ is an integral part of the quantized radiation field formalism. After the beam splitter, the quantum state of the light is in a superposition of traveling in two directions

$$
\left|\psi_{f}\right\rangle=\frac{1}{\sqrt{2}}\left(|1\rangle_{R}|0\rangle_{D}+i|0\rangle_{R}|1\rangle_{D}\right)
$$

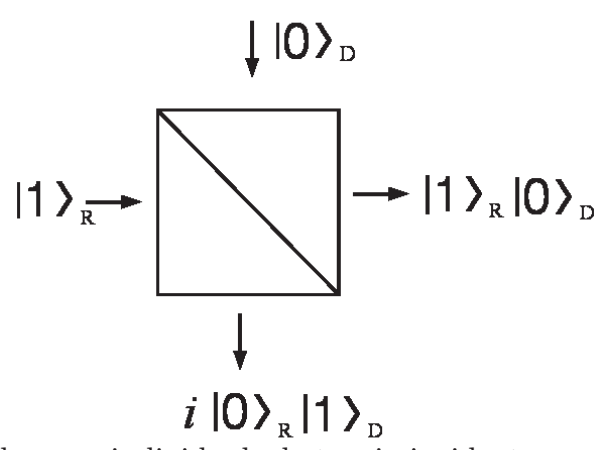

Figure 1. The outcome possibilities when an individual photon is incident on a beam splitter. If the beam splitter is $50: 50$, then the probability of the photon to be transmitted or reflected is $50 \%$.

A phase of $\pi / 2$ is introduced in the state of the reflected light as is necessary to conserve energy. ${ }^{11}$ From Eq. 2 the entering photon has a 50\% probability of being transmitted, and a $50 \%$ probability of being reflected. If a detector is placed in each of the exits the wavefunction collapses and there will be an anti-correlation, which means that either the photon is detected on transmission or the photon is detected on reflection. ${ }^{2,5,8,9}$ That a photon is in a superposition state of two states is mathematically simple but physically hard to explain to a beginning student. We can also say that the state of the photon is uncertain but balanced because it is equally possible to detect the photon coming out either way. Feynman is particularly insightful in his explanations of this superposition. ${ }^{6}$

We have taken the more intuitive Shrödinger picture for explaining this phenomenon to the beginning student. A more rigorous and common method used in quantum optics is via the Heisenberg representation and the use of photon-number creation and anihilation operators. ${ }^{10}$ We will continue our intuitive approach for the next case.

Let us now turn to the case of two photons entering the beam splitter from two separate ports, as shown in Fig. 2. A critical element of this case is the indistinguishability of the paths taken by the two photons. The pump photon produces two photons that can arrive to the detectors in two possible ways. The indistinguishability of the possibilities makes the quantum mechanical amplitudes interfere.

The state of the light can be represented by $|1\rangle_{R}|1\rangle_{D}$. After the beam splitter the state of the light is obtained by applying the beam splitter action to each occupied mode, and the state vector results, $\left|\psi_{f}\right\rangle$ :

$$
\begin{array}{r}
\frac{1}{2}\left(|1\rangle_{R}|0\rangle_{D}+i|0\rangle_{R}|1\rangle_{D}\right)\left(|1\rangle_{D}|0\rangle_{R}+i|0\rangle_{D}|1\rangle_{R}\right) \\
=\frac{i}{\sqrt{2}}\left(|2\rangle_{R}|0\rangle_{D}+|0\rangle_{R}|2\rangle_{D}\right),
\end{array}
$$

Proc. of SPIE Vol. 9289 928908-2 


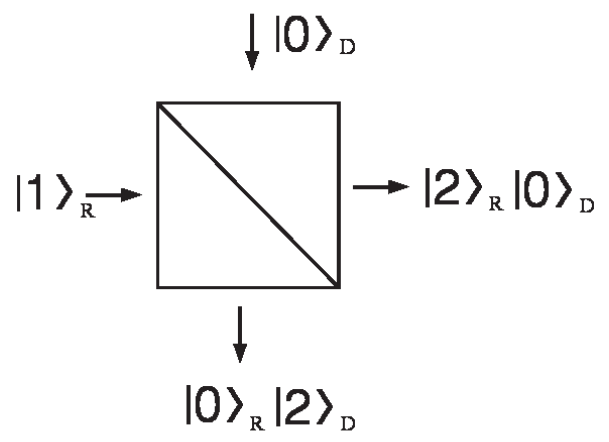

Figure 2. Two indistinguishable photons entering the beam splitter at the same time.

where we have made the condensation $|1\rangle_{R}|1\rangle_{R}=|2\rangle_{R}$, and similarly for $D$. The terms with $|1\rangle_{R}|1\rangle_{D}$ cancel out when the paths are indistinguishable. Thus, the interference is destructive. Experimentally, if we put detectors at the two output ports of the interferometer we would not get any coincidences because the destructive interference. Conversely, when the photons arrive a distinguishable times the paths are distinguishable, and so the light is in an incoherent sum of two photons each incident on the beam splitter, as in the single photon case. That situation would lead to no interference and photons heading to the two detectors (and hence, coincidences) half of the time.

This effect can be explained only with the two ways possibilities (transmitted and reflected) existence for each photon.

\section{EXPERIMENT}

The HOM experiment consists of generating a pair of photons and make them converge onto the input ports of a beam splitter. Detectors placed at the output ports of the beam splitter detect coincidences. When the photons are indistinguishable no coincidences are recorded. The photons for the experiment come from a single source: photon pairs produced simultaneously by spontaneous parametric down conversion (SPDC). In order for the photons to be indistinguishable they have to arrive at the beam splitter at the same time within the coherence time of the light, and be in the same polarization, spatial and momentum modes. The HOM experiment itself is notoriously difficult to recreate, especially in a teaching, non-research setting because of the challenge to make momentum modes of the photons overlap. That is, the photons after the beam splitter must be collinear. As a consequence, alignment is difficult and tedious. Our efforts benefited from a recent article that explains a simple way to reach the required alignment. ${ }^{12}$ The arrangement also includes a way to vary the time delay between the two photons, so to shift from the case when they are distinguishable to the case when they are indistinguishable. When the latter is achieved one observes a drop in the coincidences, the famous "HOM dip." The light is collected by lens collimators attached to multimode fibers, which send the photons to avalanche photo diode (APD) single-photon detectors.

The HOM interferometer is based in three fundamental parts: the source of the pairs of photons, an interferometer and a photon detection system. The interferometer has a dual use: is used as a interferometer (HOMI) in the experiments, and as a Mach-Zehnder interferometer $(M Z I)$ for the alignment of the optics.

The SPDC source used in the experiment is a Type I, BBO crystal which is pumped by a $25 \mathrm{~mW}$ violet laser with a $405 \mathrm{~nm}$ wave length. SPDC requires conservation of energy and momentum inside the crystal

$$
\hbar \omega_{p}=\hbar \omega_{1}+\hbar \omega_{2}
$$

and

$$
\hbar \mathbf{k}_{\mathbf{p}}=\hbar \mathbf{k}_{1}+\hbar \mathbf{k}_{\mathbf{2}}
$$

Proc. of SPIE Vol. 9289 928908-3 
It is very important for the experiment that the laser beam be parallel to the optical table so that the pair of photons is detected in the same horizontal plane. This type of source produces pairs of individual photons, with the same polarization, which follow symmetric trajectories about the pump laser beam direction, hereafter call the symmetry axis. In the degenerate case $\left(\omega_{1}=\omega_{2}\right)$, the two photons come out of the crystal with the same angle $\theta=\cos ^{-1}\left(\mathbf{k}_{\mathbf{p}} \cdot \mathbf{k}_{\mathbf{i}} /\left(k_{p} k_{i}\right), i=1,2\right.$, but at opposite sides from the symmetry axis.

The second important part of the interferometer is the alignment of the two photons through the HOMI with the aid of the MZI. The procedure is described in the next steps. First, we have to identify the angle of the photons, which in this case is $5^{\circ}$ from the symmetry axis. To do this, we use a simple procedure for alignment described in. ${ }^{3}$ This method is based on mounting the fiber collimators on two rails that pivot just below the non-linear crystal. This way the collimators always look at the crystal as the rail is moved. The rail positions are coarse adjusted to obtain the maximum number of individual photon counts at the detectors. A fine adjustment is done my maximizing the number of coincidences between the two detectors. Once this alignment is done we know an optimal paths of the down-converted photon pairs. The next step is to define these paths by placing two irises on each path, as shown in Fig. 3.

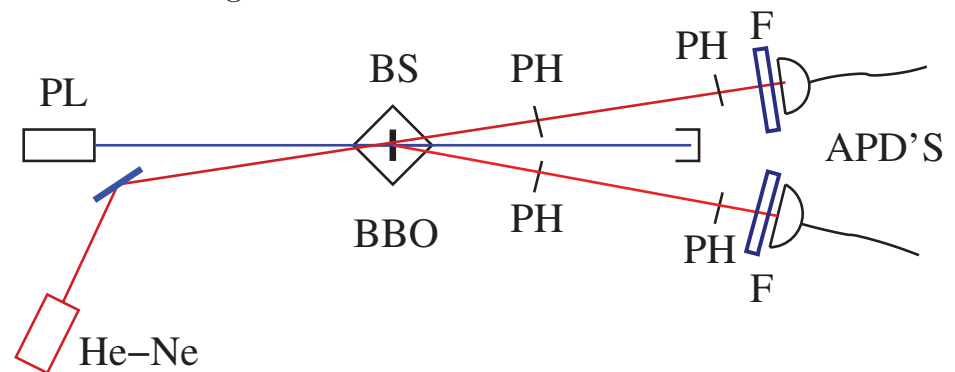

Figure 3. Schematic of the procedure to define the trajectories of the individual photons using pinholes. Afterwards, the He-Ne laser will follow these trajectories using a beam splitter to simplify the alignment. The initials used stand for: pump laser (PL), beam splitter (BS), interference filter at $810 \mathrm{~nm}(\mathrm{~F})$, and pinhole (PH).

This is used so the trajectories of the photons can be followed by a visible laser that sets the mirrors of the HOMI. The initial hardware design should allow the swapping of the non linear crystal by a beam splitter. The latter serves as the first beam splitter of the MZI, for use with the visible laser. The positions of the crystal and the beam splitter should be the same, and we should be able to swap both of them without introducing any major misalignment. In our setup we used a vertical translation stage to displace the crystal upwards, and a horizontal translation stage to displace the beam splitter. The beam splitter is positioned such that the visible laser goes through both sets of irises and thus simulating the trajectories of the two down-converted photons. The alignment is complete when the visible light incident to the collimators fully comes out of ends of the attached fibers. This is of course done with the fibers disconnected from the APD detectors, with the power to the latter being without power.

Once the visible laser is aligned to follow the trajectories of the down-converted photons the collimators and fibers can be removed to set up the hardware for the interferometer. In this setup each trajectory is to be steered by three mirrors. The mirrors are placed such that two of them are mounted in a trombone configuration, on top of a translation stage, as shown in in Fig. 4. This allows the variation of one of the length of one of the paths (lower in the figure) without altering the alignment. The 6 mirrors must be placed so that the paths mirror each other about the symmetry axis. The second beam splitter is to be placed in the intersection of the two beams. It must be able to rotate and translate in the horizontal plane so that the reflected beam and transmitted trajectories fully overlap. After the second beam splitter is in position the $M Z I$ interferometer should be ready for the next stage in the alignment.

The first step in the alignment is obtained by observing the interference fringes produced by the visible laser going through the MZI. We used a helium-neon (HeNe) laser for the visible laser. The interference pattern is easy to see because of the long coherence length of the HeNe laser beam. The alignment should proceed so that the interference fringes are thick. After this is achieved, the next step involves equalizing the two optical paths with the trombone mirror pair. We did this adjustment by driving the stage with a stepper-motor. It is 
important to use another source of light which has a very short coherence length. In our case we used light from a white fluorescent source, which had a coherence length of a few micrometers. The interferometer path length should then be adjusted carefully until a white-light interference pattern emerges. It is important to note that the observation of a white-light interference pattern implies that the difference in optical path near zero. We note that for the interferometer to be aligned, both the white light fringes and the thick guide laser fringes must be present.

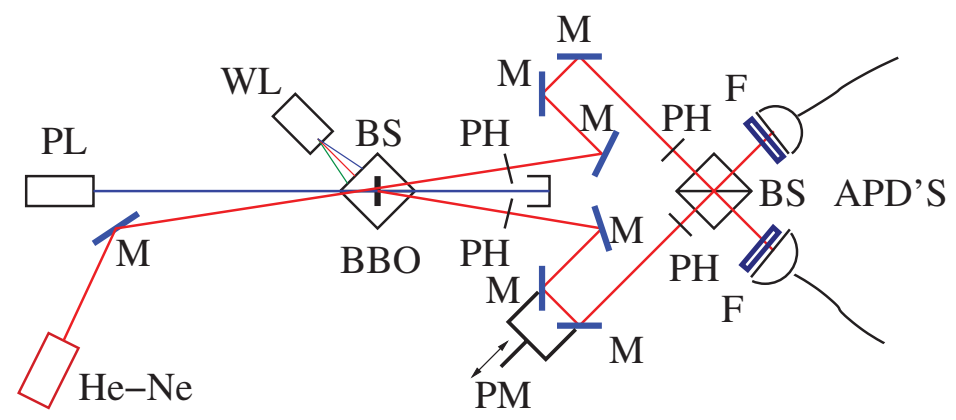

Figure 4. The figure shows the complete alignment where de He-Ne- laser helps to align the trajectories that the photons should follow. The use of the mirrors (M) helps to modify the difference in optical path using a stepper-motor $(\mathrm{PM})$. A second beam splitter is placed where the beams intersect to generate the superposition and complete the $I M Z$ interferometer. The balanced of the optical path is made by using the interference produced from a white light (WL).

After all the alignment is completed, the fiber collimators are to be placed in the trajectories of the two beams after the second beam splitter. Finally, the last step is to replace first beam splitter by the non-linear crystal, converting the MZI into the HOMI.

In the third stage of our experiment the individual photons went through a wide band filter $(810 \pm 10 \mathrm{~nm})$ before entering the collimators connected to the APD's by an optical fiber. The detectors generated square pulses that were $25 \mathrm{~ns}$ long and $3.5 \mathrm{~V}$ in height. These were then sent into ae photon-counting board. This board can detect coincidence between 4 inputs $^{13}$ within delay interval that can be adjusted to between 30 to $2500 \mathrm{~ns}$. Before taking any data, the collectors should be aligned as before, so there are a maximum number of counts of individual photons. If the system has been aligned correctly, then the number of coincidences per second in a $30 \mathrm{~ns}$ window should be really small. This is because of the destructive HOM interference. If the optical path difference $(\Delta z)$ is changed by moving the stepper-motor-driven stage, the paths may not be interfering any more, and the coincidence count will grow. There should be a minimum of coincidence counts when the destructive interference is taking place. The individual photon counts should be almost constant because the number of interfering photons is very small and it is only altered by the pairs of photons that interfere. Figure 5 shows the behavior of the photon coincidences at the output ports of the beam splitter. The average count rate of coincidences far from the interference zone is $30 \mathrm{~s}^{-1}$. A Gaussian function multiplied by a sinc function was fit to the experimental data. The fitting function depends on the bandwidth of the down-converted photons and the difference in optical path. ${ }^{14}$

\section{DISCUSSION AND CONCLUSIONS}

The HOM dip measures the coincidences of photons that come out of the beam splitter. The minimum in the coincidences embodies the destructive interference of quantum mechanical amplitudes. This interference is only explained by the simultaneously presence of the two splitting ways of the both indistinguishable photons, and then, represent the evidence of the no-locality of the quantum states. At this point the two photons travel the same distance between the crystal that generated them and the beam splitter. They enter the beam splitter at right angles, so that after the beam splitter it is not possible to know which way they arrived to the detectors. Interference disappears (i.e., the number of coincidences increases) when the the difference in optical path is changed, making the paths distinguishable. For this set of experimental parameters the width of the dip is related to the coherence length of the down-converted photons, which in our case is $40 \mu \mathrm{m}$. This compares well with our measurement: $45 \pm$. 


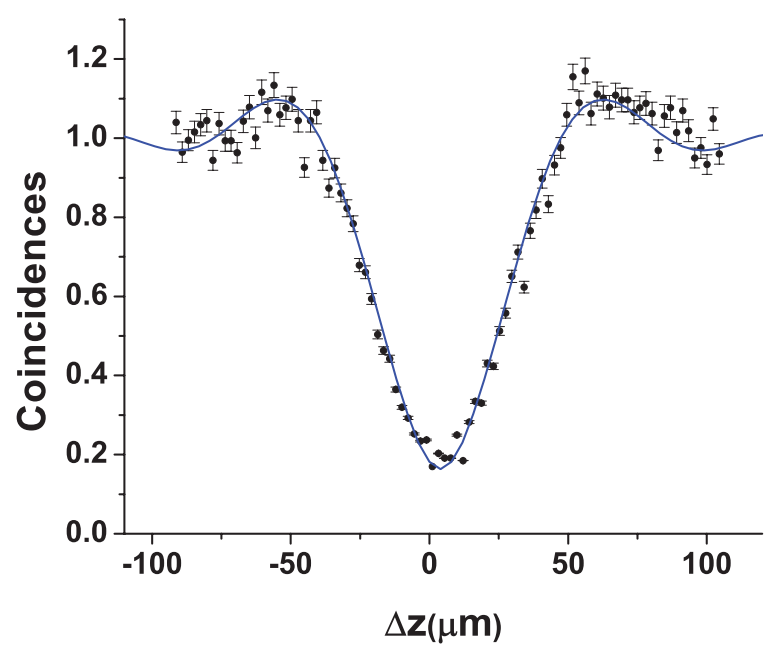

Figure 5. Normalized coincidences vs. the displacement in optical path $(\Delta z)$. Each pace produced by the motor, modifies the optical path by $2 \mu \mathrm{m}$. The average coincidence count rate outside the zone of interference is around $30^{-1}$.

The HOM interference is one of a broader class of cases involving the interference of two photons. The photons of the pair, sometimes referred to as "biphotons" can produce constructive interference, ${ }^{17}$ and may depend of a richer variety of paths in which the two photons interfere, where single-photons paths are distinguishable but two-photon paths are not. ${ }^{2,18,19}$

\section{ACKNOWLEDGMENTS}

We thank technical help, suggestions and comments from Aurora Vargas and Sergio Vergara, FCE-BUAP, This work was supported by projects UNAM DGAPA: PAPITT 117811, 117911 y CONACyT México.

\section{REFERENCES}

1. Mark Beck, Modern Undergraduate Quantum Mechanics Experiments: http://marcus.whitman.edu/ beckmk/QM/.

2. E. J. Galvez, C. H. Holbrow, M. J. Pysher, J. W. Martin, N. Courtemanche, L. Heilig, and J. Spencer, Interference with correlated photons: Five quantum mechanics experiments for undergraduates, American Journal of Physics 73, 127-140 (2005)

3. D. Dehlinger and M. W. Mitchell, Entangled photons, nonlocality, and Bell inequalities in the undergraduate laboratory, Am. J. Phys. 70, 903-910 (2002).

4. C.K.Hong,Z.Y.Ou, and L. Mandel, Measurement of subpicosecond time intervals between two photons by interference, Phys. Rev. Lett. 59, 2044 (1987).

5. P. Grangier, G. Roger, and A. Aspect, Experimental evidence for a photon anti-correlation effect on a beamsplitter., Europhys. Lett. Vol.1. Pp. 173-179, 1986.

6. Richard P. Feynman, Lectures on Physics, Vol. 3, Academic Press, 1994.

7. Jorge Carvioto-Lagos, Gustavo Armendariz P, Vctor Velzquez, Enrique Lpez-Moreno, M Grether and E J Galvez, The Hong-Ou-Mandel interferometer in the undergraduate laboratory Eur. J. Phys, 33, 1843 (2012).

8. J. J. Thorn, M. S. Neel, V. W. Donato, G. S. Bergreen, R. E. Davies, and M. Beck Observing the quantum behavior of light in an undergraduate laboratory, American Journal of Physics, 72, Issue 9, (2004) pp. 12101219 .

9. Damián San Román Alerigi La Naturaleza Cuántica de la Luz: Anticorrelación Experimental, Tesis de Licenciatura, Facultad de Ciencias-UNAM, 2009. 
10. Crhistopher C. Gerry, and Peter L. Night, Introductory Quantum Optics, Cambridge U.P. 2005.

11. C.H. Holbrow, E.J. Galvez, and M.E. Parks, Photon Quantum Mechanics and Beam Splitters, American Journal of Physics 70, 260-265 (2002).

12. P.J. Thomas, J.Y. Cheung, C.J. Chunnilall, and M.H. Dunn, The Homg-Ou-Mandel Intergerometer: A New Procedure for Alignement, Review of Scientific Instruments 80, 036101 (2009).

13. Sergio Vergara, and Aurora Vargas, Laboratorio de Robótica, Facultad de Ciencias de la Elctrónica BUAP, México.

14. Z.Y. Jeff Ou, Multi-Photon Quantum Interference, Springer 2006.

15. Leonard Mandel and Emil Wolf, Optical Coherence and Quantum Optics, Cambridge U.P. 1995.

16. Marlan O. Scully, and M. Suhail Zubairy, Quantum Optics, Cambridge U.P. 1997.

17. P.G. Kwiat, A.M. Steinberg and R.Y. Chiao, Observation of a 'quantum eraser: A revival of coherence in a two-photon interference experiment, Phys. Rev. A 45, 7729 (1992).

18. J.D. Franson, Bell inequality for position and time, Phys. Rev. Lett. 62, 2205-2208 (1989).

19. T.B. Pittman, D.V. Strekalov, A. Migdall, M.H. Rubin, A.V. Sergienko, and Y.H. Shih, Can two-photon interference be considered the interference of two photons?, Phys. Rev. Lett. 77, 1917-1920 (1996). 